\title{
DIFFERENCE SCHEMES OF GENERALIZED SOLUTIONS FOR A CLASS OF ELLIPTIC NON-LINEAR DIFFERENTIAL EQUATIONS
}

\author{
HOANG DINH DUNG
}

\begin{abstract}
It is known (see [1], [2], etc.) that in many applied problems the data are nonregular. The approximate methods for the problems of nonlinear differential equations with data belonging the Sobolev spaces $W_{p}^{l}(G)$ are presented in [3-5]. In this paper the finite-difference schemes of generalized solutions for a class of elliptic nonlinear differential equations are considered. The theorem for the convergence of approximate solution to generalized one and error norm estimations is proved in the class of equations with the right-hand side defined by a continuous linear functional in $W_{2}^{(-l)}(G)$.
\end{abstract}

Tóm tắt. Nhiều bài toán thực tiễn được dẫn về giải các bài toán đối với phương trình vi phân riêng với dũ̃ kiện không tron (xem [10], [2]). Phương pháp xấp xỉ giải một số bài toán đối vói các phương trình vi phân phi tuyến vó́i vế phải thuộc các lóp hàm khả tích khác nhau (các không gian Sobolev $W_{p}^{l}(G)$ ) được nghiên cúu trong các công trình [3-5]. Bài này xét lược đồ sai phân, nghiên cúu sự hội tụ và đánh giá sai số của nghiệm bài toán đối với một lóp phương trình vi phân phi tuyến loại ellip với vế phải không trơn độ cao kiểu các phiếm hàm tuyến tính liên tục (các không gian $W_{2}^{(-l)}(G)$ ).

\section{INTRODUCTION}

Let $G$ be a rectangle with the boundary $\partial G$. Consider the following problem

$$
\Delta u+T\left(x, u, \frac{\partial u}{\partial x_{1}}, \frac{\partial u}{\partial x_{2}}\right)=-f(x), x \in \partial, \quad u(x)=0, x \in \partial G
$$

where the given $f(x) \in W_{2}^{-l}(G)$ - the space of continuous linear functionals on the space $\stackrel{\circ}{W}_{2}^{l}(G), l$ being a nonegative integer, the function $T(x, a), a=\left(a_{0}, a_{1}, a_{2}\right)$, satisfies the conditions:

$$
\begin{aligned}
& {[T(x, a)-T(x, b)]\left(a_{0}-b_{0}\right) \geq C_{1} \sum_{i=0}^{2}\left(a_{i}-b_{i}\right)^{2},} \\
& |T(x, a)-T(x, b)| \leq C_{2}\left[\sum_{i=0}^{2}\left(a_{i}-b_{i}\right)^{2}\right]^{1 / 2},
\end{aligned}
$$

where $C_{j}, j=1,2$, are the positive constants (see [3, chap. 3, sec. 4]).

We shall use the same notations as in [6]. Consider the generalized solution $u(x)$ of the problem (1) in the space $\stackrel{\circ}{W}_{2}^{1}(G)$ satisfying the following equality:

$$
P(u, v)=\iint_{G}\left[\Delta u+T\left(x, u, \frac{\partial u}{\partial x_{1}}, \frac{\partial u}{\partial x_{2}}\right)\right] v(x) d x=-\iint_{G} f(x) v(x) d x,
$$

where $v(x)$ is a function in the space $D(G)$ of Schwartz basic functions [7].

One has $v(x) \in \stackrel{\circ}{W}_{2}^{1}(G)$. Then, by [3] (chap. 3, sec. 4), if the function $T\left(x, u, \frac{\partial u}{\partial x_{1}} \frac{\partial u}{\partial x_{2}}\right)$ satisfies the conditions $(2), f(x) \in L_{2}(G)$, there exists uniquely a solution of integral equation $(3) u(x) \in$ $\stackrel{\circ}{W} \underset{2}{1}(G) \cap W_{2}^{2}(G)$.

\footnotetext{
* This work is partially supported by the National Basics Research Program in Natural Sciences
} 


\section{CONSTRUCTION OF DIFFERENCE SCHEMES}

We first consider the case where $f(x) \in L_{2}(G)$ and let $G$ be the unit square $G=\left\{x=\left(x_{1}, x_{2}\right)\right.$ : $\left.0<x_{n}<1, n=1,2\right\}$.

Let us introduce in the region $G$ a grid $\bar{\omega}$ with interior and boundary gridpoints denoted by $\omega$ and $\gamma$ respectively $[6]$.

To construct the difference schemes one may take the test functions $v(x)$ in the form:

$$
v(x)= \begin{cases}\frac{1}{4 \pi h_{1}^{k} h_{2}^{k}} \exp \left\{-\frac{|x|^{2}}{4 h_{1}^{k} h_{2}^{k}}\right\}, & x \in e, \\ 0, & x \in \bar{G} \backslash e,\end{cases}
$$

where $e=e(x) \equiv\left\{\zeta=\left(\zeta_{1}, \zeta_{2}\right):\left|\zeta_{n}-x_{n}\right|<0,5 h_{n}, n=1,2\right\}, h_{n}$ being the steplengths, $k$ being a natural number.

Let every gridpoint $x \in \omega$ be corresponding to a mesh $e(x)$. The generalized solution (denoted by the GS) $u(x)$ of the problem (1) in e satisfies the following integral equation:

$$
\begin{aligned}
P^{e}(u, \alpha) & =\frac{1}{h_{1} h_{2}} \int_{x_{1}-0,5 h_{1} x_{2}-0,5 h_{2}}^{x_{1}+0,5 h_{1}} \int_{x_{2}+0.5 h_{2}}\left[\Delta u(\zeta)+T\left(\zeta, u, u(\zeta), \frac{\partial u}{\partial \zeta_{1}}, \frac{\partial u}{\partial \zeta_{2}}\right)\right] \alpha(\zeta) d \zeta \\
& =-R f, \quad x \in \omega,
\end{aligned}
$$

where $\alpha(\varsigma) \equiv h_{1} h_{2} v(\varsigma)$,

$$
R f \equiv \frac{1}{h_{1} h_{2}} \iint_{e} f(\varsigma) \alpha(\varsigma) d \zeta
$$

One may rewrite the equation (5) as follows

$$
\begin{aligned}
P^{e}(u, \alpha)= & \sum_{i=1}^{2} \frac{1}{h_{i}} S_{3-i}\left[\left(\alpha \frac{\partial u}{\partial x_{i}}\right)^{\left(+0,5_{i}\right)}-\left(\alpha \frac{\partial u}{\partial x_{i}}\right)^{\left(-0,5_{i}\right)}\right]-S_{1} S_{2} \sum_{i=1}^{2} \frac{\partial \alpha}{\partial \zeta_{i}} \frac{\partial u}{\partial \zeta_{i}} \\
& +S_{1} S_{2}\left[\alpha(\zeta) T\left(\zeta, u(\zeta), \frac{\partial u}{\partial \zeta_{1}}, \frac{\partial u}{\partial \zeta_{2}}\right)\right]=-R f, \quad x \in \omega,
\end{aligned}
$$

where

$$
S_{i} u(x)=\frac{1}{h_{i}} \int_{x_{1}-0,5 h_{i}}^{x_{i}+0,5 h_{i}} u\left(x_{1}, \ldots, \zeta_{i}, \ldots, x_{n}\right) d \varsigma_{i}, u^{\left( \pm 0,5_{i}\right)}(x)=u\left(x_{1}, \ldots, x_{i} \pm 0,5 h_{i}, \ldots, x_{n}\right) .
$$

Now, to obtain the difference schemes of the operator $(7) P^{e}(u, \alpha)$ one may approximate the mean integral operators $S_{i}$ by the quadrature formula of average rectangles and the partial derivatives by difference quotients as in [6] (see 2.1). Hence, one get the following difference approximations corresponding to (7), (3):

$$
\begin{aligned}
K(\breve{y}) \equiv{ }^{1} P_{1}^{e}(\breve{y}, \alpha) & =\sum_{i=1}^{2}\left(\alpha_{i} \breve{y}_{\bar{x}_{i}}\right)_{x_{i}}-S_{1} S_{2} \sum_{i=1}^{2} \alpha_{\bar{x}_{i}}(x) \breve{y}_{\bar{x}_{i}}+S_{1} S_{2} \alpha(\zeta) T\left(\zeta, \breve{y}(x), \breve{y}_{\bar{x}_{1}}, \breve{y}_{\bar{x}_{2}}\right)=-\varphi, x \in \omega, \\
\breve{y}(x) & =0, x \in \gamma
\end{aligned}
$$

and (cf. [3, chap. 3, sec. 4])

$$
\begin{aligned}
L(\widehat{y}) \equiv{ }^{2} P_{h}^{e}(\widehat{y}, \alpha) & =\sum_{i=1}^{2} \widehat{y}_{\bar{x}_{i} x_{i}}+S_{1} S_{2} \alpha(\varsigma) T\left(\varsigma, \widehat{y}(x), \widehat{y}_{\bar{x}_{1}}, \widehat{y}_{\bar{x}_{2}}\right)=-\varphi, x \in \omega, \\
\widehat{y}(x) & =0, x \in \gamma,
\end{aligned}
$$


where

$$
\begin{aligned}
u_{x_{i}} & =\frac{1}{h_{i}}\left[u^{\left(+1_{i}\right)}-u\right], u_{\bar{x}_{i}}=\frac{1}{h_{i}}\left[u-u^{\left(-1_{i}\right)}\right], \\
u^{\left( \pm 1_{i}\right)} & \equiv u^{( \pm 1, i)}(x)=u\left(x_{1}, \ldots, x_{i} \pm h_{i}, \ldots, x_{n}\right), i \geq 1, \\
\alpha_{i} & =\alpha^{\left(-0,5_{i}\right)}(x), \varphi=R f .
\end{aligned}
$$

Note that by [3] (see chap. 3, sec.4) there exists uniquely a solution of the operator equation ${ }^{2} P_{h}^{e}(y, \alpha)=-\varphi$ and, then, of the equation ${ }^{1} P_{h}^{e}(y, \alpha)$.

\section{ESTIMATION OF THE CONVERGENCE RATE}

Estimate now the method error and the approximate one of the scheme (8) and (9).

3.1. Consider the difference scheme (9) with $\varphi$ defined by (10), (7). Denote the method error by $z=\widehat{y}-u$, where $\widehat{y}$ being the solution of the problem (9). It follows from (9) that.

$$
L z=-\psi(x), x \in \omega ; z(x)=0, x \in \gamma,
$$

where $\psi(x)$ is the approximation error of the scheme (9):

$$
\Psi(x)=\varphi+L u .
$$

From (10), (7) and by formulas (10), (11) in [6, sec. 2], for the sufficiently small mesh sizes $h_{1}$ and $h_{2}$, one has

$$
\begin{aligned}
\varphi= & -\sum_{i=1}^{2}\left[S_{3-i}\left(\alpha \frac{\partial u}{\partial x_{i}}\right)^{-\left(0,5_{i}\right)}\right]_{x_{i}}+S_{1} S_{2}\left(\sum_{i=1}^{2} \frac{\partial \alpha}{\partial \zeta_{i}} \frac{\partial u}{\partial \zeta_{i}}\right) \\
& -S_{1} S_{2} T\left(\zeta, u(\zeta), \frac{\partial u}{\partial \zeta_{1}}, \frac{\partial u}{\partial \zeta_{2}}\right), x \in \omega .
\end{aligned}
$$

Thus,

$$
\begin{aligned}
\Psi= & \sum_{i=1}^{2}\left[u_{\bar{x}_{i}}-S_{3-i}\left(\alpha \frac{\partial u}{\partial x_{i}}\right)^{\left.-0,5_{i}\right)}\right]_{x_{i}}+S_{1} S_{2} \sum_{i=1}^{2} \frac{\partial \alpha}{\partial \zeta_{1}} \frac{\partial u}{\partial \zeta_{i}} \\
& -S_{1} S_{2}\left[T\left(\zeta, u(\zeta), \frac{\partial u}{\partial \zeta_{1}}, \frac{\partial u}{\partial \zeta_{2}}\right)-T\left(\varsigma, u(x), u_{\bar{x}_{1}}(x), u_{\bar{x}_{2}}(x)\right)\right]
\end{aligned}
$$

By (9) one has

$$
L_{0} \widehat{y} \equiv \sum_{i=1}^{2} \widehat{y}_{\bar{x}_{i} x_{i}}=-S_{1} S_{2}\left[T\left(\varsigma, \widehat{y}(x), \widehat{y}_{\bar{x}_{1}}, \widehat{y}_{\bar{x}_{2}}\right)\right]-\varphi \equiv \varphi_{0}, \quad x \in \omega .
$$

Then,

$$
L_{0} x=L_{0} \widehat{y}-L_{0} u \equiv-\Psi_{0}(x), x \in \omega ; z(x)=0, x \in \gamma .
$$

From (12) - (14) it follows that

$$
\begin{aligned}
\Psi_{0}=L_{0} u-\varphi_{0}= & \sum_{i=1}^{2} u_{\bar{x}_{i} x_{i}}-\sum_{i=1}^{2}\left[S_{3-i}\left(\alpha \frac{\partial u}{\partial x_{i}}\right)^{\left(-0,5_{i}\right)}\right]_{x_{i}}+S_{1} S_{2} \sum_{i=1}^{2} \frac{\partial \alpha}{\partial \zeta_{i}} \frac{\partial u}{\partial \zeta_{i}} \\
& +S_{1} S_{2} T\left(\varsigma, \widehat{y}(x), \widehat{y}_{\bar{x}_{1}}, \widehat{y}_{\bar{x}_{2}}\right)-S_{1} S_{2} T\left(\zeta, u(\zeta), \frac{\partial u}{\partial \zeta_{1}}, \frac{\partial u}{\partial \zeta_{2}}\right) .
\end{aligned}
$$

Hence,

$$
-L_{0} z=-\sum_{i=1}^{2} z_{\bar{x}_{i} x_{i}}=\sum_{i=1}^{2}\left(\eta_{i}\right)_{x_{i}}+\lambda_{0}+\beta_{0}, x \in \omega
$$


where

$$
\begin{aligned}
\eta_{i}= & u_{x_{i}}-S_{3-i}\left(\alpha \frac{\partial u}{\partial x_{i}}\right)^{\left(-0,5_{i}\right)} \\
\lambda_{0}= & S_{1} S_{2} \sum_{i=1}^{2} \frac{\partial \alpha}{\partial \zeta_{i}} \frac{\partial u}{\partial \zeta_{i}} \\
\beta_{0}= & -S_{1} S_{2}\left[T\left(\zeta, u(\zeta), \frac{\partial u}{\partial \zeta_{1}}, \frac{\partial u}{\partial \zeta 2}\right)-T\left(\zeta, u(x), u_{\bar{x}_{1}}, u_{\bar{x}_{2}}\right)\right] \\
& -S_{1} S_{2}\left[T\left(\zeta, u(\zeta), u_{\bar{x}_{1}}, u_{\bar{x}_{2}}\right)-T\left(\zeta, \widehat{y}(x), \widehat{y}_{\bar{x}_{1}}, \widehat{y}_{\bar{x}_{2}}\right)\right] \equiv \beta_{0}^{1}+\beta_{0}^{2} .
\end{aligned}
$$

Now, to obtain a priori estimation, let us scalar multiply both sides of (15) by $z(x)$ :

$$
-\sum_{i=1}^{2}\left(z_{\bar{x}_{i} x_{\imath}}, z\right)=\sum_{i=1}^{2}\left(\eta_{i_{x_{\imath}}}, z\right)+\left(\lambda_{0}, z\right)+\left(\beta_{0}, z\right)
$$

where $(a, b)$ is the scalar product on the set of net functions:

$$
(a, b)=\sum_{x \in \omega} a(x) b(x) h_{1} h_{2} .
$$

Since $z(x)=0$ for $x \in \gamma$, one has

$$
\sum_{i=1}^{2}\left\|z_{\bar{x}_{i}}\right\|_{i}^{2} \equiv\|\nabla z\|_{0, \omega}^{2} \leq \sum_{i=1}^{2}\left\|\eta_{i}\right\|_{i}\left\|z_{\bar{x}_{i}}\right\|_{i}+\left(\left\|\lambda_{0}\right\|+\left\|\beta_{0}\right\|\right)\|z\|,
$$

where

$$
\begin{aligned}
\left\|z_{\bar{x}_{i}}\right\|_{i}^{2} & \equiv\left(z_{\overline{x_{i}}}, z_{\bar{x}_{i}}\right]_{i}, \\
\left(a,\left.z\right|_{1}\right. & =\sum_{y_{1}=1}^{N_{1}} \sum_{j_{2}=1}^{N_{2}-1} a\left(j_{1} h_{1}, j_{2} h_{2}\right) z\left(j_{1} h_{1}, j_{2} h_{2}\right) h_{1} h_{2} \\
\left(a,\left.z\right|_{2}\right. & =\sum_{y_{1}=1}^{N_{1}-1} \sum_{j_{2}=1}^{N_{2}} a\left(j_{1} h_{1}, j_{2} h_{2}\right) z\left(j_{1} h_{1}, j_{2} h_{2}\right) h_{1} h_{2} \\
\|a\|^{2} & =\sum_{y_{1}=1}^{N_{1}} \sum_{j_{2}=1}^{N_{2}} a^{2}\left(j_{1} h_{1}, j_{2} h_{2}\right) h_{1} h_{2} .
\end{aligned}
$$

Then

$$
\|z\|_{1, \omega} \leq C\left(\left\|\eta_{1}\right\|_{1}+\left\|\eta_{2}\right\|_{2}+\left\|\lambda_{0}\right\|+\left\|\beta_{0}\right\|\right)
$$

where the constant $C$ is independent of $h\left(|h|^{2}=h_{1}^{2}+h_{2}^{2}\right)$ and $z(x)$,

$$
\|z\|_{1, \omega}^{2} \equiv\|z\|_{0, \omega}^{2}+\|\nabla z\|^{2},\|z\|_{0, \omega} \equiv\|z\| \text {. }
$$

Now, we first consider the functional $\eta_{1}(x)$ defined by $(16)$ :

$$
\eta_{1}(x)=u_{x_{1}}-\frac{1}{h_{2}} \int_{x_{2}-0,5 h_{2}}^{x_{2}+0,5 h_{2}} \alpha\left(x_{1}-0,5 h_{1}, \zeta_{2}\right) \frac{\partial u}{\partial \zeta_{1}}\left(x_{1}-0,5 h_{1}, \zeta_{2}\right) d \zeta_{2} .
$$

This expression coincides with the one of $\eta_{1}(x)(19)$ in [6]. Hence, by (23) in [6] we have

$$
\left|\eta_{1}(x)\right| \leq M|h|\left(h_{1} h_{2}\right)^{-\frac{1}{2}}\|u\|_{2, e^{1}},
$$

where $e^{1}$ is the following mesh of the grid $\omega$ : 


$$
\begin{aligned}
e^{i}=e^{i}(x) & \equiv\left\{\xi=\left(\zeta_{1}, \zeta_{2}\right): x_{i}-h_{i}<\zeta_{i}<x_{i},\left|\zeta_{3-i}-x_{3-i}\right|<0,5 h_{3-i}\right\}, \\
\|u\|_{m, e^{1}} & \equiv\|u\|_{W_{2}^{m}\left(e^{1}\right)}=\left(\sum_{|\alpha| \leq m_{e}} \int_{e^{1}}\left|D^{\alpha} u\right|^{2} d x\right)^{1 / 2} .
\end{aligned}
$$

The functional $\eta_{2}(x)$ is estimated similarly. Then,

$$
\left\|\eta_{i}\right\|_{i} \leq C|h|\left(\sum_{x}\|u\|_{2, e^{i}}^{2}\right)^{1 / 2} \leq C|h|\|u\|_{2,(i} .
$$

The expression of $\lambda_{0}$ coincides with the one of $\eta_{0}(15)$ in [6]. Then, by (26) in [6] we have

$$
\left\|\lambda_{0}\right\| \leq C|h||H(h)|\|u\|_{1,(\text { r }}
$$

where $H(h) \rightarrow 0$ as $h_{1}, h_{2} \rightarrow 0$.

Consider now $\beta_{0}^{1}$ in (18). The difference of the form $\beta_{0}^{1}$ is estimated in [3] (see chap. 3, sec. 4), one has

$$
\left\|\beta_{0}^{1}\right\| \leq C|h|\|u\|_{2, G} .
$$

From the last inequality and (16) it follows that

$$
\left\|\beta_{0}\right\| \leq C|h|\|u\|_{2,(\text { r }} .
$$

Finally, combining (18) - (21) we get

$$
\|z\|_{1, \omega}=\|\widehat{y}-u\|_{1, \omega} \leq C|h|\|u\|_{2, G} .
$$

3.2. Consider the following difference scheme

$$
M y=\frac{1}{2}(K+L) y=-\varphi, x \in \omega ; y(x)=0, x \in \gamma,
$$

where $y=\frac{1}{2}(\breve{y}+\widehat{y}), \breve{y}$ and $\widehat{y}$ are defined (8) and (9) respectively. Then,

$$
\begin{aligned}
M y= & \frac{1}{2} \sum_{i=1}^{2}\left[\left(1+\alpha_{i}\right) y_{\bar{x}_{i}}\right]_{x_{i}}-\frac{1}{2} S_{1} S_{2} \sum_{i=1}^{2} \alpha_{\bar{x}_{i}} y_{\bar{x}_{i}}+ \\
& +\frac{1}{2} S_{1} S_{2}\left[\alpha(\zeta) T\left(\zeta, y(x), y_{\bar{x}_{1}}, y_{\bar{x}_{2}}\right)+T\left(\zeta, y(x), y_{\bar{x}_{i}}, y_{\bar{x}_{2}}\right)\right] \\
= & -\varphi, x \in \omega \\
y(x)= & 0, x \in \gamma .
\end{aligned}
$$

Thus,

$$
\begin{aligned}
M_{0} y & \equiv \sum_{i=1}^{2}\left[\left(1+\alpha_{i}\right) y_{\bar{x}_{i}}\right]_{x_{i}} \\
& =S_{1} S_{2} \sum_{i=1}^{2} \alpha_{\bar{x}_{i}, y_{\bar{x}_{i}}}-S_{1} S_{2}\left[\alpha T\left(\zeta, y(x), y_{\bar{x}_{1}}, y_{\bar{x}_{2}}\right)+T\left(\zeta, y(x), y_{\bar{x}_{1}}, y \bar{x}_{2}\right)\right]-2 \varphi \\
& \equiv \varphi_{0}, \quad x \in \omega, \\
y(x) & =0, x \in \gamma .
\end{aligned}
$$

From the last equality, (7) and (12) one has

$$
M_{0} z=\varphi_{0}-M_{0} u=-\Psi(x), x \in \omega ; \quad z(x)=0, x \in \gamma
$$

where $z=y-u$ is the method error, 


$$
\begin{aligned}
\Psi(x) & =-\sum_{i=1}^{2}\left[\left(1+\alpha_{i}\right) z_{\bar{x}_{i}}\right]_{x_{i}}=\sum_{i=1}^{2}\left[\eta_{i}+\mu_{i}\right]_{x_{i}}+\lambda_{0}+\beta_{0}+q_{0} \\
\eta_{i} & =u_{x_{i}}-S_{3-i}\left(\alpha \frac{\partial u}{\partial x_{i}}\right)^{\left(-0,5_{i}\right)}, \mu_{i}=\alpha_{i}=\alpha_{i} u_{x_{i}}-S_{3-i}\left(\alpha \frac{\partial u}{\partial x_{i}}\right)^{\left(-0,5_{i}\right)} \\
\lambda_{0} & =S_{1} S_{2} \sum_{i=1}^{2}\left[2 \frac{\partial \alpha}{\partial \zeta_{i}} \frac{\partial u}{\partial \zeta_{i}}-\alpha \bar{x}_{i} y_{\bar{x}_{i}}\right] \\
\beta_{0} & =-S_{1} S_{2}\left[T\left(\zeta, u(\zeta), \frac{\partial u}{\partial \zeta_{1}}, \frac{\partial u}{\partial \zeta_{2}}\right)-T\left(\zeta, y, y_{\bar{x}_{1}}, y_{\bar{x}_{2}}\right)\right] \\
q_{0} & =-S_{1} S_{2} \alpha(\zeta)\left[T\left(\zeta, u(\zeta), \frac{\partial u}{\partial \zeta_{1}}, \frac{\partial u}{\partial \zeta_{2}}\right)-T\left(\zeta, y, y_{\bar{x}_{1}}, y_{\bar{x}_{2}}\right)\right]
\end{aligned}
$$

By (24), (25), in the same way as in 3.1 one has

$$
\|z\|_{1, \omega} \leq C\left(\sum_{i=1}^{2}\left(\left\|\eta_{i}\right\|_{i}+\left\|\mu_{i}\right\|_{i}\right)+\left\|\lambda_{0}\right\|+\left\|\beta_{0}\right\|+\left\|q_{0}\right\|\right) .
$$

In (26) $\eta_{i}$ has the form (16), then one has the estimation (19) for $\eta_{i}$.

The expression of $\mu_{i}$ coincides with the one of $\gamma_{i}$ (31) in [6], then by (39) in [6] one has

$$
\left\|\mu_{i}\right\| \leq C|h|^{m-1}\left|H_{i}(h)\right|\|u\|_{m,(r}, \quad m=2,3,
$$

where $H_{i}(h), i=1,2$ tend to zero as $h \rightarrow 0$.

$\lambda_{0}$ has the form (33) of $\tilde{\gamma}_{0}$ in [6], then by (44) in [6],

$$
\left\|\lambda_{0}\right\| \leq C|h||H(h)|\|u\|_{2,(r} .
$$

$\beta_{0}$ has the form (16), then by (21) one has

$$
\left\|\beta_{0}\right\| \leq C|h|\|u\|_{2,(;}
$$

Consider the last summand $q_{0}$ in (26). The form of $q_{0}$ is analogous to $\beta_{0}$ and one may easily verify that

$$
\left\|q_{0}\right\| \leq C|h||H(h)|\|u\|_{2, G} .
$$

Now, combining (19), (2) - (30) yields

$$
\|\breve{y}+\widehat{y}-2 u\|_{1, \omega} \leq C \mid h^{m-1}\|u\|_{m, G}, \quad m=2,3 .
$$

Finally, by (22) and (31) we get the estimation of method error for the difference scheme (8):

$$
\|\breve{y}-u\|_{1, \omega} \leq C|h|^{m-1}\|u\|_{m, G}, \quad m=2,3 .
$$

Remark. In a manner analogous to the proof of the inequalities (22) and (32), one may verify that these inequalities are also valid if in the formula of the GS $u(x)(5),(7), v(x)\left(=\frac{\alpha(x)}{h_{1} h_{2}}\right)$ is a Schwartz basic function.

3.3. The estimates (22) and (32) are obtained with the assumption $f \in L_{2}(G)$, now we show that the results may be generalized to the equations with right-hand side $f \in W_{2}^{(-l)}(G), W_{2}^{(-l)}(G)$ being the space of continuous linear functionals on the space $\stackrel{\circ}{W}_{2}^{l}(G), l$ is a nonegative integer. For example, $f$ is the Dirac delta function $\delta$.

Indeed, by our assumption, $f(x) \in D^{\prime}(G), D^{\prime}(G)$ being the space of Schwartz distributions. Therefore, by the theorem on local structure of the distributions (see [7, chap. 3, sec. 6]) there exists a function $g(x) \in L_{\infty}(e)$ and an integer $k \geq 0$ such that 


$$
f(x)=D_{1}^{k} \ldots D_{n}^{k} g(x)
$$

where $x \in e$, the set $e$ is compact in $G \in R^{n}, D_{i} \equiv \partial / \partial x_{i}$.

Let $v(x) \in D(e)$, By (5) and (33) one has

$$
\iint_{e}\left[\Delta u(x)+T\left(x, u, \frac{\partial u}{\partial x_{1}}, \frac{\partial u}{\partial x_{2}}\right)\right] v(x) d x=-\iint_{e} g(x) \tilde{v}(x) d x,
$$

where

$$
\tilde{v}(x)=D_{1}^{k} D_{2}^{k} v(x)(n=2) .
$$

We see that $\tilde{v}(x)$ is also a test function: $\tilde{v}(x) \in D(e) \subset \stackrel{\circ}{W}_{2}^{l}(e)$ and $g(x) \in L_{2}(e)$. Thus, the equation (34) has the form (5). Hence, one may repeat the procedure used above for the difference schemes (8), (9) and obtaines the following.

Theorem. Let in the problem (1) the function $T($.$) satisfy the conditions (2) and the right-hand$ side $f \in W_{2}^{(-l)}(G)$. Then the solution y of the difference scheme (8) or (9) ( $y=\breve{y}$ or $\left.\widehat{y}\right)$ converges to the GS (5) u(x) of the problem (1) in the grid norm $W_{2}^{1}(\omega)$ with the rate $O(|h|)$, that is, one has the following error estimation

$$
\|y-u\|_{1, \omega} \leq C|h|\|u\|_{2,(\dot{*}}
$$

where the constant $C$ is independent of $h$ and $u(x)$.

\section{REFERENCES}

[1] G. I. Marchuk, Mathematical Modelling in the Environment Problems, Nauka, Moscow, 1982 (Russian).

[2] V.S. Vladimirov, Generalized Functions in Mathematical Physics, Mir, Moscow, 1979.

[3] A. A. Samarskii, R. D. Lazarov, V. I. Makarov, Difference Schemes for Generalized Solutions of Differential Equations, Vus. Univ., Moscow, 1987.

[4] C. Padra, A posterior error estimators for nonconforming approximation of some quasi-Newtonian flows, SIAM J. Numer. Anal. 34 (4) (1997) 1600-1615.

[5] C. N. Davson, M. F. Wheeler, C.S. Woodward, A two-grid finite difference scheme for non-linear parabolic equations, SIAM J. Numer. Anal. 35 (2) (1998) 435-452.

[6] Hoang Dinh Dung, Difference schemes for generalized solutions of some elliptic differential equations, I, Journal of Computer Science and Cybernetics 15 (1) (1999) 49-61.

[7] L. Schwartz, Théorie des Distributions, Hermann, Paris, 1978.

Institute of Mathematics, NCST of Vietnam

Received March 20, 2000 Revised January 5, 2001 